\title{
Structure of the activated ROQ1 resistosome directly recognizing the pathogen effector XopQ
}

\author{
$\underline{\text { Raoul Olivier Martin }}{ }^{1,3,4}$, Tiancong $\mathbf{Q i}^{2,3,4}$, Haibo Zhang ${ }^{2}$, Furong Liu ${ }^{3,4}$, Miles King ${ }^{3,4}$, Claire Toth ${ }^{5}$, Eva Nogales ${ }^{5,6,7}$, \\ Brian Staskawicz ${ }^{3,4}$ \\ ${ }^{1}$ Biophysics Graduate Group, University of California, Berkeley, CA 94720, USA; \\ ${ }^{2}$ Center for Plant Biology, School of Life Sciences, Tsinghua University, Beijing 100084, China.; \\ ${ }^{3}$ Department of Plant and Microbial Biology, University of California, Berkeley, CA 94720 USA; \\ ${ }^{4}$ Innovative Genomics Institute, University of California, Berkeley, CA 94720 USA.; \\ ${ }^{5}$ Department of Molecular and Cellular Biology, University of California, Berkeley, CA 94720, USA; \\ ${ }^{6}$ Howard Hughes Medical Institute, University of California, Berkeley, CA 94720, USA.; \\ ${ }^{7}$ Molecular Biophysics and Integrated Bioimaging Division, Lawrence Berkeley National Laboratory, University of California, \\ Berkeley, CA 94720, USA \\ raoulmartin@berkeley.edu
}

Plants and animals detect pathogen infection via intracellular nucleotide-binding leucine-rich repeat receptors (NLRs) that directly or indirectly recognize pathogen effectors and activate an immune response. How effector sensing triggers NLR activation remains poorly understood. Structure-function studies of these complexes are hampered by low levels in native tissue, our inability to express them recombinantly, and their instability in solution. We overcame sample limitation problems and solved a $3.8 \AA$ resolution cryo-EM structure of the activated ROQ1, an NLR native to N. benthamiana with a Toll-like interleukin-1 receptor (TIR) domain, bound to the Xanthomonas effector XopQ. ROQ1 directly binds to both the predicted active site and surface residues of XopQ while forming a tetrameric resistosome that brings together the TIR domains for downstream immune signaling. Our results suggest a mechanism for the direct recognition of effectors by NLRs leading to the oligomerization-dependent activation of a plant resistosome and signaling by the TIR domain.

Keywords: Innate immunity, Cryo-electron microscopy, pathogen detection 